\title{
Perfil Clínico-epidemiológico de Crianças e Adolescentes com Câncer em um Serviço de Oncologia
}

doi: https://doi.org/10.32635/2176-9745.RBC.2018v64n3.26

Clinical and Epidemiological Profile of Children and Adolescentes with Cancer in an Oncology Service
Perfil Clínico Epidemiológico de Niños y Adolescentes con Cáncer en un Servicio de Oncología

Cintia Flôres Mutti'; Vanessa Gomes da Cruz ${ }^{2}$; Leidiene Ferreira Santos ${ }^{3}$; Daiana de Araújo ${ }^{4}$; Silvana Bastos Cogo ${ }^{5}$ Eliane Tatsch Neves ${ }^{6}$

Resumo

Introduçáo: $\mathrm{O}$ progresso no controle do câncer nos últimos 50 anos é inegável; entretanto, no Brasil, ele ainda representa a segunda causa de morte na população infantojuvenil, e estudos não têm evidenciado declínio desses dados. Objetivo: Analisar o perfil clínico-epidemiológico de crianças e adolescentes com câncer em um Serviço de Oncologia. Método: Estudo documental retrospectivo, com busca de dados em prontuários de crianças e adolescentes, com diagnóstico de câncer no período de 2008 a 2014 . Os dados foram coletados no período de maio a agosto de 2015, armazenados em banco de dados informatizado e submetidos à análise estatística descritiva. Este estudo foi aprovado pelo Comitê de Ética em Pesquisa da Universidade Federal do Tocantins (protocolo 116/2014). Resultados: A idade média ao diagnóstico foi de 8 anos, sendo a maioria do sexo masculino; e os tipos de câncer mais frequentes foram as leucemias, linfomas e os tumores do sistema nervoso central. O uso da quimioterapia predominou entre os tratamentos utilizados. Referente à situação-desfecho, obtiveram alta por cura (11\%); em tratamento (14\%); acompanhamento pós-tratamento (31\%); e foram a óbito (29\%). Conclusáo: $\mathrm{O}$ perfil de crianças e adolescentes com câncer deste estudo denotou a complexidade e a dimensão biopsicossocial, que envolveram a terapêutica do câncer infantojuvenil, traduzidas pelo número expressivo de internaçóes hospitalares e óbitos em crianças menores de 5 anos. Recomenda-se a capacitação dos profissionais de saúde para a realizaçáo do diagnótico precoce e para a excelência do tratamento multiprofissional em busca não só da cura, mas também da qualidade de vida.

Palavras-chave: Neoplasias; Criança; Adolescente; Epidemiologia.

\begin{abstract}
Introduction: Progress in cancer control in the last 50 years is undeniable, however, in Brazil it still represents the second cause of death in the child and adolescent population and studies have not shown a decline in this data. Objective: To analyze the clinical-epidemiological profile of children and adolescents with cancer in an Oncology Service. Method: Retrospective documentary study, with search of data in medical records of children and adolescents, with diagnosis of cancer in the period from 2008 to 2014. Data were collected from May to August 2015, stored in a computerized database and submitted to statistical analysis descriptive. This study was approved by the Research Ethics Committee of the Federal University of Tocantins (protocol 116/2014). Results: The mean age at diagnosis was 8 years, the majority of which were male and the most frequent types of cancer were Leukemia, Lymphomas and Central Nervous System Tumors. The use of chemotherapy predominated among the treatments used. Regarding the situation-outcome, they were discharged for cure (11\%); in treatment $(14 \%)$; post-treatment follow-up (31\%) and died (29\%). Conclusion: The profile of children and adolescents with cancer in this study denoted the complexity and biopsychosocial dimension that involved the treatment of childhood and adolescence cancer, translated by the expressive number of hospital admissions and deaths in children under 5 years of age. It is recommended that health professionals be trained to perform the early diagnosis and for the excellence of multi-professional treatment, seeking not only cure but also quality of life. Key words: Neoplasms; Child; Adolescent; Epidemiology.
\end{abstract}

\section{Resumen}

Introducción: el progreso en el control del cáncer en los últimos 50 años es innegable, sin embargo, en Brasil todavía representa la segunda causa de muerte en la población infantojuvenil y los estudios no han evidenciado la declinación de esos datos. Objetivo: analizar el perfil clínico-epidemiológico de niños y adolescentes con cáncer en un Servicio de Oncología. Método: estudio documental retrospectivo, con búsqueda de datos en prontuarios de niños y adolescentes, con diagnóstico de cáncer en el período de 2008 a 2014. Los datos fueron recolectados en el período de mayo a agosto de 2015, almacenados en banco de datos informatizado y sometidos al análisis estadístico descriptivo. Este estudio fue aprobado por el Comité de Ética en Investigación de la Universidad Federal de Tocantins (protocolo 116/2014). Resultados: La edad media al diagnóstico fue de 8 ańos, siendo la mayoría del sexo masculino y los tipos de cáncer más frecuentes fueron las Leucemias, Linfomas y los Tumores del Sistema Nervioso Central. El uso de la quimioterapia predominó entre los tratamientos utilizados. En cuanto a la situación de desencadenamiento, se obtuvieron altas por cura (11\%); en tratamiento (14\%); seguimiento post-tratamiento $(31 \%)$ y fueron a muerte (29\%). Conclusión: El perfil de nińos y adolescentes con cáncer de este estudio denotó la complejidad y la dimensión biopsicosocial que involucraron la terapéutica del cáncer infantojuvenil traducidas por el número expresivo de internaciones hospitalarias y muertes en niños menores de 5 años. Se recomienda la capacitación de los profesionales de salud para la realización del diagnóstico precoz y para la excelencia del tratamiento multiprofesional, en busca no sólo de la cura, sino también de la calidad de vida.

Palabras clave: Neoplasias; Niño; Adolescente; Epidemiología.

\footnotetext{
${ }^{1}$ Universidade Federal de Tocantins (UFT). Palmas (TO), Brasil. Orcid iD: https://orcid.org/0000-0003-0437-2568

2 Fundação Escola de Saúde Pública de Palmas (Fesp). Palmas (TO), Brasil. Orcid iD: https://orcid.org/0000-0002-0903-1159

${ }^{3}$ UFT. Palmas (TO), Brasil. Orcid ID: https://orcid.org/0000-0002-2969-6203

${ }^{4}$ Universidade Federal de Santa Maria (UFSM). Santa Maria (RS), Brasil. Orcid iD: https://orcid.org/0000-0003-4296-023X

${ }^{5}$ UFSM. Santa Maria (RS), Brasil. Orcid iD: http://orcid.org/0000-0002-1686-8459

${ }^{6}$ UFSM. Santa Maria (RS), Brasil. Orcid iD: https://orcid.org/0000-0002-1559-9533

Endereço para correspondência: Cintia Flôres Mutti. Rua Appel, 65, apto. 53 - Nossa Senhora de Fátima. Santa Maria (RS), Brasil. CEP 97015-030. E-mail: cintiamutti02@gmail.com.
} 


\section{INTRODUÇÃO}

De acordo com a Organização Mundial da Saúde (OMS), em 2012, houve, em todo o mundo, 14,1 milhóes de casos novos de câncer e 8,2 milhóes de mortes por esse agravo. Estima-se que, em 2030, os números mundiais serão de 23,6 milhôes de novos casos da doença. Enquanto isso, no território brasileiro, a estimativa, para o biênio 2016-2017, foi de 600 mil novos casos de câncer com altas taxas de incidência e mortalidade ${ }^{1,2}$

Assim como os demais tipos, o câncer infantojuvenil tem como definição um grupo de doenças caracterizado pela multiplicação desordenada de células atípicas e de ocorrência em qualquer local do corpo ${ }^{3}$. É considerado um espectro de diferentes neoplasias malignas, que variam segundo o tipo histológico, localização de origem da doença, sexo, idade e raça. Essas neoplasias diferem dos tumores dos adultos quanto aos seus aspectos morfológicos, ao comportamento clínico e às localizaçôes primárias e, por isso, devem ser estudadas separadamente ${ }^{4}$. Tratam-se de diferenças relacionadas ao prognóstico, aspectos histológicos e localização do tumor. As neoplasias de maior incidência na infância são as leucemias, as do sistema nervoso central (SNC) e os linfomas. Para os adultos, a incidência é maior para o câncer de pele do tipo não melanoma, tumores de próstata e cânceres de mama ${ }^{1}$.

O Instituto Nacional de Câncer José Alencar Gomes da Silva (INCA) estima que, no Brasil, para cada ano do biênio 2018-2019, ocorrerão 420 mil casos novos de câncer, sem considerar o câncer de pele náo melanoma. $\mathrm{O}$ percentual dos tumores infantojuvenis brasileiros é de 3\%. Assim sendo, ocorrerão 12.500 casos novos de câncer em crianças e adolescentes até os 19 anos. As Regiōes Sudeste e Nordeste apresentarão os maiores números de casos novos, 5.300 e 2.900 , respectivamente, seguidas pelas Regiōes Centro-Oeste com 1.800, Sul 1.300, e Norte com 1.2005.

Destaca-se que, no país, o câncer respondeu pela oitava posição entre as causas de óbito em crianças de 0 a 4 anos, e foi a principal causa de morte na faixa etária de 5 a 19 anos em 2014, de acordo com o Sistema de Informação de Mortalidade do Ministério da Saúde (SIM) ${ }^{6}$. Dados referentes à mortalidade por câncer na população dos Estados Unidos e Argentina vão ao encontro dos dados brasileiros, sendo a segunda causa de mortalidade em crianças de 0 a 14 anos e entre 5 a 14 anos de idade, respectivamente, superados apenas pelas causas externas ${ }^{3,6}$.

O diagnóstico do câncer infantil, as constantes internaçôes e os tratamentos dolorosos modificam a rotina da criança e de sua família, constituindo um desafio à equipe de saúde multiprofissional ${ }^{7}$. Nesse contexto, a detecção precoce do câncer infantil, assim como o rápido início do tratamento, possui significativa importância para o prognóstico da criança, estando diretamente relacionada com o bom desenvolvimento da terapêutica e resultados positivos, como a cura do câncer ${ }^{8}$.

Entretanto, o diagnóstico em estágios iniciais é complexo. Comumente, a criança apresenta sinais e sintomas inespecíficos, que estão presentes em outras doenças mais frequentes na infância, como febre, vômitos, emagrecimento, sangramentos, adenomegalias generalizadas, dor óssea generalizada e palidez?

Desse modo, é indispensável o desenvolvimento de uma assistência qualificada, visando ao diagnóstico precoce e ao tratamento em tempo oportuno. De acordo com estudos em países desenvolvidos, avanços no diagnóstico clínico e biológico, uso de terapias adequadas ao risco e otimização dos cuidados de suporte resultam em aumento dramático nas taxas de cura de crianças com câncer ${ }^{10,11}$.

No entanto, pontua-se que, em várias regiōes brasileiras, a curabilidade do câncer infantil está comprometida, especialmente pelo déficit de hospitais especializados em oncologia pediátrica, pela falta de médicos especialistas e de diagnóstico tardio da doença na rede de atençáo básica ${ }^{12}$.

Considera-se que conhecer a realidade e o contexto de uma população, assim como os dados relativos à incidência e as particularidades do câncer no país ou em determinadas áreas da federação, pode ser instrumento de suporte para planejar e acompanhar as açóes de saúde, almejando impactar de maneira positiva e concreta em açôes preventivas e assistenciais ${ }^{13}$. Esta pesquisa objetivou analisar o perfil clínico-epidemiológico de crianças e adolescentes com câncer em um Serviço de Oncologia.

\section{MÉTODO}

Estudo documental retrospectivo, realizado a partir de dados registrados nos prontuários de crianças e adolescentes atendidos, no período de março de 2008 a dezembro de 2014, na unidade de oncologia de um hospital público localizado na Região Norte do Brasil. Esse serviço é referência para o Estado do Tocantins e áreas circunvizinhas, como o Sul do Pará, do Maranhão e Bahia. O referido hospital possui administração estadual, prestando assistência hospitalar e ambulatorial, com nível de atenção de média e alta complexidades ${ }^{14}$.

A coleta de dados ocorreu na própria unidade de saúde, nos meses de maio a agosto de 2015. Para identificar os prontuários das crianças e adolescentes, foi consultada planilha eletrônica, cedida pelo serviço administrativo da unidade de oncologia, em que constavam as seguintes variáveis analisadas nesta pesquisa: nome, idade, sexo, procedência, acompanhante, tipo de câncer, número de internações, desfecho e óbitos. 
Para a organização das informações constantes nos prontuários, considerando os objetivos desse estudo, elaborou-se um instrumento próprio de coleta de dados, contendo itens relacionados às características sociodemográficas e clínicas das crianças e adolescentes. Os registros foram armazenados em um banco informatizado e submetidos à análise estatística descritiva, apresentados por meio de frequência simples e percentuais.

Para categorizar os tipos de câncer analisados no estudo, foi considerada a Classificação Internacional do Câncer na Infância de 1996 (CICI-3), realizada de acordo com o tipo morfológico ${ }^{15}$.

Ao total, foram analisados 160 prontuários, considerando os critérios de seleção: prontuários em que constavam a confirmação diagnóstica para câncer e idade entre 0 a 18 anos incompletos. Foram excluídos aqueles que não continham informaçóes mínimas, tais como, idade e diagnóstico da doença, totalizando 14 prontuários.

Esta pesquisa foi aprovada pelo Núcleo de Ensino e Pesquisa da instituição hospitalar e pelo Comitê de Ética em Pesquisa da Universidade Federal do Tocantins (UFT), sob o número de parecer 116/2014.

\section{RESULTADOS}

De acordo com os prontuários analisados, somente 39 $(24,4 \%)$ crianças e adolescentes residiam na mesma cidade da unidade de saúde. Identificou-se, assim, que a maioria das famílias, muitas vezes, precisava viajar longas distâncias para conseguir tratamento oncológico. Em alguns casos, advindos de cidades localizadas no Estado do Pará e Maranhão, eram percorridos mais de mil quilômetros até a unidade de oncologia do hospital público pesquisado.

De acordo com os prontuários analisados, houve maior incidência de câncer em crianças com até 5 anos de idade $(54 ; 36,97 \%)$, e predominância no sexo masculino (94;64\%). A idade média ao início do tratamento foi 8,11 anos (Tabela 1).

Tabela 1. Características sociodemográficas de crianças e adolescentes com câncer, atendidos na unidade de oncologia de um hospital público, Região Norte, Brasil, 2015 (N=146)

\begin{tabular}{c|c|c}
\hline \multicolumn{1}{c|}{ Variáveis } & $\mathbf{f}$ & $\%$ \\
\hline $\begin{array}{c}\text { Idade (anos) } \\
\leq 1 \text { ano }\end{array}$ & 14 & 9,58 \\
\hline $2 \mathrm{e} \leq 5$ & 40 & 27,39 \\
\hline $6 \mathrm{e} \leq 9$ & 36 & 24,65 \\
\hline $10 \mathrm{e}<12$ & 14 & 9,58 \\
\hline $12 \mathrm{e}<15$ & 20 & 13,69 \\
\hline $15 \mathrm{e} \leq 18$ & 22 & 15,06 \\
\hline Sexo & & \\
\hline Feminino & 52 & 35,6 \\
\hline Masculino & 94 & 64,4 \\
\hline
\end{tabular}

Consideraram-se o CICI- $3^{15}$, os tipos de câncer infantojuvenis identificados neste estudo como, predominantemente, as leucemias (69; 47,26\%), seguidas pelos linfomas (27; 18,49\%) (Tabela 2).

Tabela 2. Distribuição dos tipos de câncer em crianças e adolescentes com diagnóstico de câncer em um serviço de oncologia, período de 2008 a 2014, Brasil, 2015 ( $N=146)$

\begin{tabular}{l|c|c}
\hline \multicolumn{1}{c}{ Tipos de câncer } & f & $\%$ \\
\hline Leucemias & 54 & 36,98 \\
\hline Leucemia linfoide aguda & 10 & 6,84 \\
\hline Leucemia mieloide aguda & 3 & 2,05 \\
\hline Leucemia mieloide crônica & 2 & 1,36 \\
\hline Leucemia bifenotípica & 13 & 8,90 \\
\hline Linfomas & 7 & 4,79 \\
\hline Linfoma de Hodgkin & 4 & 2,73 \\
\hline Linfoma de Burkitt & 3 & 2,05 \\
\hline Linfoma não Hodgkin &
\end{tabular}

Sistema nervoso central e neoplasias

intracranianas e intraespinhais

\begin{tabular}{l|l|l}
\hline Ependimomas & 5 & 3,42 \\
\hline Meduloblastoma & 4 & 2,73 \\
\hline Astrocitoma & 3 & 2,05 \\
\hline Tumor neuroectodérmico primitivo & 2 & 1,36 \\
\hline Outros gliomas & 4 & 2,73 \\
\hline $\begin{array}{l}\text { Tumores do sistema nervoso } \\
\text { simpático }\end{array}$ & $\mathbf{6}$ & $\mathbf{4 , 1 0}$ \\
\hline Neuroblastoma & 6 & 4,10 \\
\hline Tumores renais & $\mathbf{3}$ & $\mathbf{2 , 0 5}$ \\
\hline Tumor de Wilms & 3 & 2,05 \\
\hline Tumores hepáticos & $\mathbf{2}$ & $\mathbf{1 , 3 6}$ \\
\hline Hepatoblastoma & 1 & 0,68 \\
\hline Hepatocarcinoma & 1 & 0,68 \\
\hline Tumores ósseos malignos & $\mathbf{5}$ & $\mathbf{3 , 4 2}$ \\
\hline Osteossarcoma & 5 & 3,42 \\
\hline Sarcomas de partes moles & $\mathbf{7}$ & $\mathbf{4 , 7 9}$ \\
\hline Rabdomiossarcoma & 5 & 3,42 \\
\hline Sarcoma alveolar de partes moles & 1 & 0,68 \\
\hline Tumor de partes moles & 1 & 0,68 \\
\hline Tumores de células germinativas, & $\mathbf{4}$ & $\mathbf{2 , 7 3}$ \\
\hline trofoblásticas e gonodais & 2 & 1,36 \\
\hline Tumor de saco vitelínico & 1 & 0,68 \\
\hline Coriocarcinoma metastático & 1 & 0,68 \\
\hline Carcinoma de ovário & $\mathbf{5}$ & $\mathbf{3 , 4 2}$ \\
\hline Outros carcinomas & &
\end{tabular}

Em relação aos tipos de tratamentos, identificou-se que em 65 (45\%) foi usada unicamente quimioterapia e em 56 (38\%) ela esteve associada a outras intervenções, tais como, radioterapia $(25 ; 17 \%)$, cirurgia $(22 ; 15 \%)$, transplante de células-tronco hematopoiéticas (3;2\%), e radioterapia e cirurgia (6;4\%). Destaca-se que, quando foi 
necessário, as crianças/adolescentes foram encaminhadas para os serviços de radioterapia e transplante de células-tronco hematopoiéticas de outros centros especializados.

Dos 146 casos analisados, 106 (72,60\%) necessitaram de internação hospitalar, sendo $70(47,94 \%)$ em unidade de internação; 7 (0,47\%) em unidade de terapia intensiva somente (UTI); e $29(19,86 \%)$ em ambas. Ao somar-se o quantitativo de internaçôes hospitalares, obteve-se o resultado de 1.051, sendo 1.007 em unidade de internaçáa e 44, em UTI, no período de aproximadamente de sete anos. Além disso, entre aqueles que necessitaram de tratamento em unidade hospitalar, o número de internaçóes variou de 1 a 28 vezes; enquanto, entre os que necessitaram de tratamento em UTI, o número de internaçôes variou de 1 a 4 vezes. Em 116 (72,5\%) casos, a mãe foi apontada como acompanhante principal.

Os resultados apontaram, ainda, que, ao término da coleta de dados, houve 16 (11\%) casos de cura; 46 (31\%) encontravam-se em acompanhamento pós-tratamento; $20(14 \%)$ se mantinham em tratamento; três $(2 \%)$ em cuidados paliativos; quatro (3\%) foram transferidos para outro serviço de oncologia; quatro $(3 \%)$ estavam com tratamento interrompido e 43 evoluíram a óbito (29\%). Em relação aos quatro casos de tratamento interrompido, três ocorreram em razão da falta de profissional de saúde especializado, e um da gestação da adolescente. Foram registrados seis $(3,7 \%)$ casos de doença metastática (Gráfico 1).

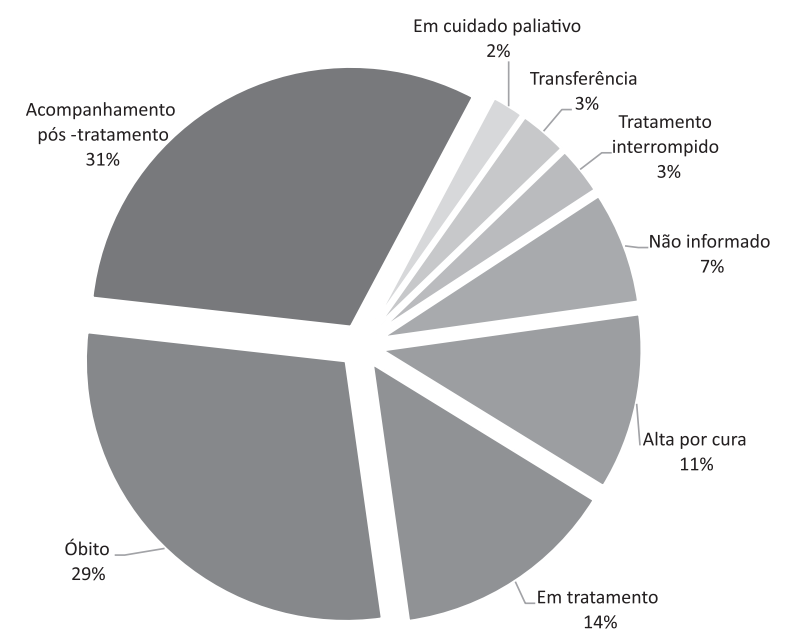

Gráfico 1. Caracterização dos desfechos de crianças e adolescentes com diagnóstico de câncer em um serviço de oncologia, no período de 2008 a 2014, Brasil, 2015

Foram evidenciados, ainda, o predomínio de óbitos em crianças do sexo masculino $(69,76 \%)$ e o diagnóstico de leucemias $(21 ; 48,83 \%)$ na faixa etária de 2 a 5 anos de idade (Tabela 3 ).
Tabela 3. Caracterização dos casos de óbito de crianças e adolescentes com diagnóstico de câncer em um serviço de oncologia, no período de 2008 a 2014, Brasil, 2015 ( N=43)

\begin{tabular}{l|c|c}
\hline \multicolumn{1}{c|}{ Características } & f & $\%$ \\
\hline Idade (anos) & & \\
\hline$\leq 1$ & 2 & 4,65 \\
\hline $2 \leq 5$ & 10 & 23,25 \\
\hline $6 \leq 9$ & 7 & 16,27 \\
\hline $10<12$ & 4 & 9,30 \\
\hline $12<15$ & 9 & 20,93 \\
\hline $15 \leq 18$ & 7 & 16,27 \\
\hline NR & 4 & 9,20 \\
\hline Sexo & & \\
\hline Feminino & 13 & 30,23 \\
\hline Masculino & 30 & 69,76 \\
\hline Tipo de câncer & & \\
\hline Leucemias & 21 & 48,83 \\
\hline Tumores do sistema nervoso central & 6 & 13,95 \\
\hline Linfomas & 5 & 11,62 \\
\hline Neuroblastoma & 3 & 6,97 \\
\hline Tumores ósseos & 2 & 4,65 \\
\hline Tumores de partes moles & 2 & 4,65 \\
\hline Tumores renais & 2 & 4,65 \\
\hline Tumores hepáticos & 1 & 2,32 \\
\hline Outros & 1 & 2,32 \\
\hline & &
\end{tabular}

\section{DISCUSSÃO}

Os achados deste estudo constataram uma predominância do câncer em crianças e adolescentes do sexo masculino, o que se assemelharam a resultados apontados em outras pesquisas realizadas no Brasil e em outros países, como Estados Unidos, Colômbia e Bolívia $^{13,16-19}$.

Pesquisa realizada sobre a incidência destes tumores nos Estados Unidos apontou como sendo maior entre indivíduos do sexo masculino do que no sexo feminino, identificando, ainda, que adolescentes de 15 a 19 anos foram mais acometidos por neoplasias do que crianças compreendidas entre 0 a 14 anos $^{19}$.

Corroborando esses estudos, outro realizado em serviços de oncologia no Brasil, nos Estados do Rio Grande do Sul e Piauí, também apontou predomínio do sexo masculino e evidenciou que a maior parte dos indivíduos acometidos por tais neoplasias foi de crianças menores de 10 anos $^{18}$. Os resultados no serviço de oncologia pesquisado aproximaram-se dos estudos brasileiros, citados acima, sendo identificado que a maioria dos casos também ocorreu em menores de 10 anos de idade.

Informaçóes obtidas por meio do perfil clínico e sociodemográfico de determinada populaçáo, como o tipo e localização do tumor, extensão da doença, idade, nível de 
escolaridade dos pais e distância dos centros de tratamento são fatores que podem interferir no tempo gasto, desde o início da apresentação dos primeiros sintomas até o diagnóstico do câncer em crianças e adolescentes ${ }^{20}$.

A avaliação da incidência, da mortalidade e da morbidade hospitalar é importante para o conhecimento do perfil de câncer, e a efetiva vigilância para a ampla utilização das informaçóes, a fim de que estas se transformem em açôes efetivas para o controle do câncer em crianças, adolescentes e adultos jovens ${ }^{21}$.

Os tipos de tumores infantojuvenis que predominaram na pesquisa foram leucemias, seguidas por linfomas e tumores do SNC. Dessa forma, é possível observar que os resultados apresentados evidenciaram as leucemias, em especial a leucemia linfocítica aguda, como as neoplasias que mais acometeram indivíduos menores de 19 anos, sendo o resultado compatível com as demais pesquisas apresentadas, tanto no Brasil quanto no exterior ${ }^{13,16-18,22}$.

Enquanto, nos Estados Unidos, os tipos de cânceres infantojuvenis mais evidentes foram as leucemias, seguidas por tumores do SNC e linfomas ${ }^{19}$. Em Cali, Colômbia, a ordem observada foi a de leucemias, tumores do SNC, linfomas e sarcomas de tecidos moles ${ }^{19}$. Já, em Cochabamba, na Bolívia, encontrou-se a sequência de leucemias, retinoblastomas e linfomas ${ }^{17}$.

A realidade indicada por estudos realizados em serviços de oncologia no Brasil apresentou, também, uma predominância das leucemias e uma variação entre os demais tipos de câncer. No Estado do Paraná, a ordem de ocorrência foi a de leucemias, seguidas pelos linfomas, retinoblastomas e tumores do SNC. No Espírito Santo, as leucemias foram seguidas por linfomas, tumores do SNC e tumores renais ${ }^{23}$; e, no Piauí, as leucemias foram seguidas por linfomas, tumores ósseos, tumores renais e tumores do $\mathrm{SNC}^{18}$.

O presente estudo, assim como a pesquisa realizada no Estado do Paraná, apontou a quimioterapia como a terapêutica mais utilizada entre os pacientes oncológicos infantis ${ }^{24}$, sendo utilizada de maneira isolada ou associada a outros tratamentos. A quimioterapia traz aos familiares da criança sentimentos opostos e conflitantes. Ou seja, ao mesmo tempo em que se evidenciam o medo e tristeza, devido aos efeitos colaterais ocasionados pela terapêutica, também se observam a esperança e a expectativa da cura, mediante a utilizaçáo do tratamento ${ }^{25}$.

Notou-se que a radioterapia utilizada pelos pacientes contemplados no estudo foi sempre associada a outras terapêuticas, como quimioterapia e/ou cirurgia. No que diz respeito à radioterapia, a literatura apontou que esta deve ser utilizada com cautela e que, cada vez menos, vem sendo utilizada em tratamentos de crianças e adolescentes, em virtude dos efeitos tardios ocasionados por ela ${ }^{20}$.
As práticas terapêuticas utilizadas no tratamento do câncer, assim como qualquer outro tratamento, possuem efeitos colaterais. Sabe-se que o tratamento do câncer possui toxidades hematológicas, gastrointestinais, dermatológicas, podendo, ainda, ocorrer, em casos de uso de quimioterápicos e complicaçôes infecciosas, que são a causa principal de morbidade e mortalidade de crianças e adolescentes imunocomprometidos em tratamento oncológico ${ }^{20}$.

Crianças acometidas por doenças crônicas, geralmente, necessitam de hospitalização por períodos prolongados e frequentes internaçôes, que são responsáveis pela alteração da rotina da criança, assim como também por acarretar sentimentos de medo, angústia, amadurecimento precoce e impor limitações para tal indivíduo ${ }^{26}$. Os efeitos da hospitalização ultrapassam a doença e acabam ocasionando mudanças no cotidiano e na estrutura familiar ${ }^{8}$.

Entre os resultados dessa pesquisa, os dados mostraram a figura materna como a principal acompanhante durante o tratamento, participando tanto de forma isolada quanto em conjunto com demais familiares envolvidos e/ou terceiros. Fato este que se assemelha a outros estudos que abordaram a temática familiar da criança diagnosticada com câncer ${ }^{23,25-28}$.

O câncer infantojuvenil traz consigo uma sobrecarga e tensão, sendo que as necessidades básicas do acompanhante da criança/adolescente, como higiene pessoal, alimentação e sono, são alteradas e sofrem prejuízos, tendo em vista que o familiar direciona sua atenção e cuidado integral à criança/adolescente ${ }^{29}$.

$\mathrm{O}$ achado que se destacou neste estudo foi o elevado número de óbitos, $29 \%$ dos participantes, sendo que tais óbitos ocorreram mais em crianças do que adolescentes, e mais entre indivíduos do sexo masculino do que feminino. Em outro estudo realizado em dois municípios da Regiáo Nordeste do Brasil, houve aumento das taxas de mortalidade ${ }^{7}$. Esses dados corroboraram os dados da pesquisa que identificou que a mortalidade por cânceres infantojuvenis aumentou no Norte e Nordeste de 2\% a $3 \%$ ao ano; e, nas Regiôes Sul, Sudeste e Centro-Oeste, reduziu de $0,5 \%$ a $1,5 \%$ ao ano $^{30}$.

Em outras pesquisas ambientadas no Brasil, os pacientes que foram a óbito, predominantemente, possuíam o diagnóstico de leucemia, tumor do $\mathrm{SNC}$ ou linfoma ${ }^{7,13,24,31}$. Analisando a faixa etária dos pacientes que foram a óbito, foi possível identificar que este estudo se contrapôs ao resultado de uma pesquisa realizada no Estado do Paraná, onde foi demonstrado que adolescentes apresentaram um risco três vezes maior de morte em comparação com as crianças ${ }^{31}$.

Entre os fatores associados ao aumento e ao número expressivo de mortalidade em Estados do Norte e Nordeste 
do Brasil, destacaram-se questóes socioeconômicas, o sistema público ou privado de saúde e a distância de centros médicos e, ainda, o número reduzido de serviços de oncologia e médicos oncologistas ${ }^{3,32}$.

Outro fator relacionado foi a demora do diagnóstico, visto que, geralmente, quanto mais avançada está a doença, menores são as chances de cura e maiores são as sequelas decorrentes do tratamento que acaba sendo mais agressivo. Desde 2011, o Ministério da Saúde vem trabalhando na implementação de protocolos que auxiliem os profissionais da rede de atenção à saúde na condução dos casos suspeitos e confirmados, dentro de uma linha de cuidado que estabeleça fluxos e ações desde a atenção básica até a alta complexidade 5 .

De acordo com um estudo realizado com profissionais da atenção primária no município de Campinas ${ }^{33}$, havia insegurança por parte dos profissionais perante esse diagnóstico, pois o reconhecimento dos sinais e sintomas do câncer infantojuvenil era complexo. Além disso, para eles, o diagnóstico precoce avançou, no sentido de não se fixar somente em uma taxonomia diagnóstica, mas também no estabelecimento da relação que ia além do médico/paciente, incluindo características subjetivas imprescindíveis ao estabelecimento de vínculo. Vale ressaltar que, aos profissionais atuantes na área da oncologia pediátrica, cabe o desafio de reduzir as consequências do tratamento oncológico, assim como estabelecer um cuidado que contemple o paciente e a família de forma integral ${ }^{34}$.

As limitações do estudo foram a impossibilidade de identificar o tempo entre os primeiros sinais e sintomas, diagnóstico e início do tratamento, presença de outros agravos à saúde, disponibilidade de medicamentos e possíveis associações entre o número de óbitos e outras variáveis. Isso, por tratar-se de um estudo documental, em que somente foram analisadas informaçóes constantes em prontuários.

Nesse sentido, é importante que os profissionais de saúde sejam capacitados para realizarem o preenchimento adequado dos prontuários, haja vista que as informaçóes podem contribuir para traçar medidas preventivas e de tratamento.

\section{CONCLUSÃO}

O perfil clínico-epidemiológico de crianças e adolescentes com câncer, atendidos em um Serviço de Oncologia de um hospital público localizado na Região Norte do Brasil, indicou uma prevalência de leucemias, linfomas e tumores do SNC em crianças até 5 anos de idade e do sexo masculino.
Além disso, a complexidade e a dimensão biopsicossocial, que envolveram a terapêutica do câncer infantojuvenil, traduziram-se pelo número expressivo de internaçóes hospitalares, denotando a necessidade de uma assistência de sáude multiprofissional especializada.

Os dados obtidos no estudo estiveram em concordância com algumas pesquisas realizadas no Brasil e no exterior. Entretanto, o estudo apresentou dados alarmantes no que diz respeito à mortalidade. Ressalta-se que, no município estudado, não havia serviço de oncologia pediátrica, fato este que pôde ter contribuído para o defecho de óbitos em números significativo.

Recomenda-se a urgência na capacitaçáo dos profissionais de saúde para a realização do diagnótico precoce e o fornecimento de informaçóes à população, visando à identificação e à associação dos sinais e sintomas relacionados ao câncer infantil, assim como a importância de um atendimento especializado e/ou encaminhamento para centros especializados com a maior brevidade.

Com os achados deste estudo, pretendeu-se contribuir para o planejamento de açóes de saúde voltadas à assistência especializada a essa população e à sua família, visando ao aumento da sobrevida, da qualidade de vida e a um melhor prognóstico para esses indivíduos. Para tanto, almeja-se a busca por um tratamento multiprofissional especializado do câncer infantojuvenil, não somente buscando a cura, mas também a qualidade de vida para essas crianças e adolescentes.

\section{CONTRIBUIÇÕES}

Cintia Flôres Mutti trabalhou na concepção e planejamento do projeto de pesquisa, na obtenção e/ ou análise de dados, bem como na redação, revisão crítica do manuscrito e na aprovação final da versão para a publicação. Vanessa Gomes da Cruz trabalhou na concepção e planejamento do projeto de pesquisa e na obtenção e/ou análise de dados, bem como na redaçáo. Leidiene Ferreira Santos, Daiana de Araújo, Silvana Bastos Cogo e Eliane Tatsch Neves trabalharam na redação, revisão crítica do manuscrito e na aprovação final da versão para a publicação.

\section{DECLARAÇÃO DE CONFLITO DE INTERESSES}

Nada a declarar.

\section{FONTES DE FINANCIAMENTO}

Não há. 


\section{REFERÊNCIAS}

1. World Health Organization. International Agency for Research on Cancer; Cancer research Uk. World Cancer Factsheet. Cancer Research UK, London; 2014. [cited 2015 Dec 2]. Available from: https://www.cancerresearchuk.org/ sites/default/files/cs_report_world.pdf

2. Ministério da Saúde (BR), Secretaria de Atenção à Saúde, Departamento de Atenção Especializada e Temática. Protocolo de diagnóstico precoce para oncologia pediátrica [internet]. 2017. [acesso 2018 Jul 14]. Disponível em: http://portalarquivos2.saude. gov.br/images/pdf/2017/fevereiro/17/Protocolo-deDiagnostico-Precoce-do-Cancer-Pediatrico.pdf

3. Siegel R, Desantis C,Virgo K, Stein K, Mariotto A, Smith T, Cooper D, Gansler T, Lerro C, Fedewa S, Lin C, Leach C, Cannady RS, Cho H, Scoppa S, Hachey M, Kirch R, Jemal A, Ward E. Cancer treatment and survivorship statistics. CA Cancer J. Clin. 2012;62(4):220-41.

4. Instituto Nacional de Câncer José Alencar Gomes da Silva. Incidência, mortalidade e morbidade hospitalar por câncer em crianças, adolescentes e adultos jovens no Brasil: informaçóes dos registros de câncer do sistema de mortalidade [internet]. 2016. [acesso 2018 Mar 8]. Disponível em: http://www1.inca.gov.br/wcm/ incidencia/2017/pdf/versao-completa.pdf

5. Instituto Nacional de Câncer José Alencar Gomes da Silva. Estimativa 2018: incidência de câncer no Brasil. [Internet]. Rio de Janeiro: INCA; 2017. [acesso 2018 Jul 14]. Disponível em: http://www.inca.gov.br/ estimativa/2018/estimativa-2018.pdf

6. Ministério de Salud (ARG), Secretaria de Politicas. Regulacion e Institutos, Direccion de Estadisticas e Informacion de Salud. Estadisticas vitales: Informacion Básica - Año 2010. Buenos Aires: Ministerio de Salud; 2011. [acceso 2018 Jul 15]. Disponible: http://www. deis.gov.ar/publicaciones/archivos/serie5nro54.pdf

7. Silva LF, Cabral IE. As repercussôes do câncer sobre o brincar da criança: implicaçóes para o cuidado de enfermagem. Texto Contexto Enferm. 2014; 23(4): 935-43.

8. Fermo VV, Lourençato GN, Medeiros TS, Anders JC, Souza AIJ. O diagnóstico precoce do câncer infantojuvenil: o caminho percorrido pelas famílias. Esc Anna Nery Rev Enferm. 2014;18(1):54-9.

9. Michalowski MB, Lorea CF, Rech A, Santiago P, Lorenzoni M, Taniguchi A, et al. Diagnóstico precoce em oncologia pediátrica. Bol. Cient. Ped. 2012;1(1):13-8.

10. Hudson MM, Link MP, Simone JV. Milestones in the curability of pediatric cancers. J. Clin. Oncol. 2014;32:2391-2397.

11. Pritchard-Jones K, Pieters R, Reaman GH, Downie P, Calaminus $G$, et al. Sustaining innovation and improvement in the treatment of childhood cancer: Lessons from high-income countries. Lancet Oncol. 2013;14:e95-e103.

12. Ministério da Saúde (BR), Secretaria de Atenção à Saúde, Departamento de Atenção Especializada e Temática. Protocolo de diagnóstico precoce para oncologia pediátrica [Internet]. 2017. [acesso 2018 Jul 14]. Disponível em: http://portalarquivos2.saude. gov.br/images/pdf/2017/fevereiro/17/Protocolo-deDiagnostico-Precoce-do-Cancer-Pediatrico.pdf

13. Silva JKO, Moreira DC Filho, Mahayri N, Ferraz RO, Friestino FS. Câncer infantil: monitoramento através dos registros de câncer de base populacional. Rev Bras Cancerol. 2012;58(4):681-6.

14. Cadastro Nacional de Estabelecimentos da Saúde. Estabelecimento de Saúde [Internet]. 2014. [acesso 2014 Set 11]. Disponível em: http://cnes.datasus. gov.br/Exibe_Ficha_Estabelecimento.asp?VCo_ Unidade $=1721002786117$

15. Steliarova-Foucher E, Stiller C, Lacour B, Kaatsch P. International classification of childhood cancer, third edition. Cancer. 2005;103(7):1457-67.

16. Bravo LE, Garcia LS, Collazos P, Aristizabal P, Ramirez O. Descriptive epidemiology of childhood cancer in Cali, Colombia 1977-2011. Colomb. Med. 2013;44(3):155-64.

17. Carpio-Deheza G, Lafuente-Riverola VH, SalasMendoza BT. Análisis clínico-epidemiológico de la presentación y diagnóstico del cáncer pediátrico en Cochabamba, estúdio multi-institucional en los Hospitales Pediátricos: HNMAV y CPAP. Rev Méd Cient "Luz Vida". 2011;2(1):34-8.

18. Pedrosa AO, Lira R Filho, Santos FJL, Gomes RNS, Montes LRS, Portela NLC. Perfil clínicoepidemiológico de clientes pediátricos oncológicos atendidos em um hospital de referência do Piauí. R. Interd. 2015;8(3):12-21.

19. Siegel DA, King J, Tai E, Buchanan N, Ajani UA, LJ. Cancer incidence rates and trends among children and adolescents in the United States, 2001-2009. Pediatrics. 2014;134(4):945-55.

20. Instituto Nacional de Câncer José Alencar Gomes da Silva, Coordenação Geral de Ações Estratégicas, Coordenação de Educação. ABC do câncer: abordagens básicas para o controle do câncer. Rio de Janeiro: INCA; 2012.

21. Pérez CJ, Armando AM Niño, Zambrano GR, Amaya ICA. Cáncer infantil en el Área metropolitana de Bucaramanga, Colombia, 2003-2007. Med. UNAB. 2011;14(2):86-93.

22. Presti PF, Macedo CRD, Caran EM, Rodrigues AHD, Petrilli AS. Estudo epidemiológico de câncer na adolescência em centro de referência. Rev. Paul. Pediátr. 2012;30(2):210-6. 
23. Hadas TC, Gaete AE, Pianovski MAD. Câncer pediátrico: perfil epidemiológico dos pacientes atendidos no serviço de oncologia pediátrica do hospital de clínicas da UFPR. Rev Med UFPR. 2014;1(4):141-9.

24. Bauer DF, Ferrari RA, Reis TB, Tacla MT. Crianças com câncer: caracterização das internaçóes em um hospital escola público. Semina Ciênc Biol Saúde. 2015;36(1):9-16.

25. Kanda MH, Contim D, Gonçalves JR, Santos EA. A percepçáo dos familiares cuidadores sobre o tratamento quimioterápico em crianças e adolescentes. Cogitare Enferm. 2014;19(1):84-8.

26. Nóbrega RD, Collet N, Gomes IP, Holanda ER, Araújo YB. Criança em idade escolar hospitalizada: significado da condição crônica. Texto Contexto Enferm. 2010;19(3):425-33.

27. Duarte MLC, Zanini LN, Nedel MNB. O cotidiano dos pais de crianças com câncer e hospitalizadas. Rev Gaúcha Enferm. 2012;33(3):111-8.

28. Medeiros EGMS, Leite RFB, Ramos DKR, Almeida LAL. Repercussóes do câncer infantil no cotidiano do familiar cuidador. Rev Rene. 2014;15(2):233-9.

29. Silveira RA, Oliveira ICS. O cotidiano do familiar/ acompanhante junto da criança com doença oncológica durante a hospitalização. Rev Rene. 2011;12(3):532-9.

30. Ferman S, Santos MO, Ferreira JM, Reis RS, Oliveira JF, Pombo-de-Oliveira MS, et al. Childhood cancer mortality trends in Brazil, 1979-2008. Clinics. [Internet]. 2013 [cited 2018 Jul 20];68(2):219. Available from: http://dx.doi.org/10.6061/clinics/2013(02) OA16. Cited in: PubMed; PMID 23525319.

31. Marchi JA, Wakiuchi J, Sales CA, Mathias TAF, Fernandes CAM. Câncer infanto-juvenil: perfil de óbitos. Rev Rene. 2013;14(4):911-9

32. Rodrigues KE, Camargo B. Diagnóstico precoce do câncer infantil: responsabilidade de todos. Rev Assoc Med Bras. 2003;49(1):29-34.

33. Friestino JK; Corrêa CR; Moreira Filho DC. Percepçôes dos profissionais sobre o diagnóstico precoce do câncer infanto-juvenil na atenção primária à saúde. Rev Bras Cancerol. 2017;63(4):265-272.

34. Fermo VV, Lourençato GN, Medeiros TS, Anders JC, Souza AIJ. O diagnóstico precoce do câncer infantojuvenil: o caminho percorrido pelas famílias. Esc Anna Nery Rev Enferm. 2014;18(1):54-9. 